\title{
What Lies Behind the Mirror: An Intercultural Pragmatic Approach to Citation Practices
}

\author{
Liyin Zhang \\ School of Foreign Languages \\ Northwest University \\ Xi'an, P. R. China 710127
}

\begin{abstract}
Academic writers are set in the socio-cultural context, searching for recognition from potential readers with citations as the bridge. Appropriate citations, however, can be hard to achieve. In particular, for novice EFL writers, their lack of control over linguistic skills and insufficient mastery of academic conventions may lead to a more conscious approach. Kecskes proposed the socio-cognitive approach from the intercultural pragmatic perspective, which was adopted to analyze the citation practices conducted by three Chinese doctoral students and the results suggest that participants' citation practices could be better viewed as a privatized process of collective salience and co-constructed academic conventions, with the interplay of societal and cognitive factors as the main driving force herein.
\end{abstract}

Keywords-societal factors; individual factors; novice writers; citation practices; academic writing

\section{INTRODUCTION}

Academic writing is viewed as a situated activity mediated by institutional discourses and practices [1]. As a prominent feature of academic discourse, citation is measured as the ability to use sources effectively and appropriately, and is also an essential skill that novice writers must acquire [2], [3]. However, it may be problematic for novice writers with different linguistic and disciplinary backgrounds when they are set in an unfamiliar academic conventional system [4], [5], [6], [7], [8]

Cultural difference may be one of the most heatedly discussed factors involved. Reference [9], though in a mild way, attributes the inappropriate source use by novice ESL/EFL writers, particularly those from the Far East, to being culturally-conditioned, representing a commonly accepted view. Responsive arguments proposed by [10] indicate the unreliability of informants in Sowden's research and suggest that culture is an important yet not the only influencing factor of cross-cultural source use. Insufficient training in academic writing [11], lack of language proficiency [12] and writing experiences, among others, may be possible reasons for the inappropriateness of citations by novices. The debate on citation practices with a stress on the differences in cultural perceptions helps to better understand the developmental discourse practices and cultural notions

The research is funded by the Research Grant of Department of Education of Shaanxi Province, China (No 16JK1748). that ESL/EFL writers bring to English academic writing. Cultural difference, however, is not the mask for novices' performance problems. Writing instructors' oversensitivity to students' understanding of possible cultural differences may prevent them from effectively pointing out students' inappropriate use of sources [13].

Admittedly, novice writers may go through a rather bumpy road and carry certain distinctive features in their academic writing process. They may have limited understandings of when and how to signal the role that a source can play in a new text [2]. For example, they feel difficult to distinguish forms of plagiarism other than verbatim copying without using quotation marks and/or without giving the source [14]. At times, they may also face the dilemma of presenting their critical analysis in a mild way or in a harsh manner [12]. The case for EFL or EFL writers is even more severe.

Considering the complexity of citation itself, either a cognitive-philosophical approach or a socio-cultura approach with respective emphasis on priori and post factum sides of intention cannot fully investigate writers' actual performance. Here I adopted a socio-cognitive approach (SCA) [15], [16], viewed as the "underlying theory of intercultural pragmatics" by [16], not as the definitive insight into citation practice, but believe it can provide a vocabulary for understanding the complex issue and, to some extent, escaping the legal and ethical claims of academic dishonesty and unsuccessful ownership surrounding inappropriate citation.

This current study with a socio-cognitive approach explored the possibility of approaching the issue of citation practices by novice ESL/EFL writers, and focused on why they were adopting certain strategies in citing based on the texts and interview data collected throughout their writing process. Hopefully, the study could contribute to the application of SCA to the productive process of written communication that has not been extensively and fully covered in the relevant scholarship so far.

The theoretical base of the research design was presented and discussed at first. The main foci here are on the necessity to introduce a dynamic view towards the interplay of societal and cognitive factors involved in writers' citation practices, and on the point that citing should be regarded as a process 
in which writers build the connection between existing sources and the current research. In the subsequent section, participants' attitudes, motives and self-initiated strategies are analyzed, and their patterns are discussed. Then the interplay of societal factors and individual factors involved in participants' citation practices was examined.

The following questions guided this research.

- What features participants' citation practices in academic writing (e.g. attitudes, motives and selfinitiated strategies)?

- What are the factors that influence participants' citation practices?

- How do these factors interplay with each other?

\section{THEORETICAL BACKGROUND}

Socio-cognitive approach is popular among scholars and is applied to various issues in different disciplines, such as [17] approach to second language acquisition, [18] attempt to knowledge transfer and [19] analysis on discourse and context. In the socio-cognitive paradigm as a whole, human functioning is viewed as the product of a dynamic interplay of personal, behavioral, and environmental influences. It is to take into account both societal and individual factors including cooperation and egocentrism that are not antagonistic phenomenon in interaction [10]. SCA combines the positivist and social constructionist views of contexts and avoids a one-sided view, emphasizing the selective or the constitutive role of context.

Positivists consider the meaning of words or texts lies in the objective things they refer to. However, according to the epistemology of social constructivists, practices are the carriers of meaning [20], [21]. All knowledge, including commonsense, is derived and reinforced by social interactions. With positivist emphasizing the individual and social constructivist the collective, the privatized interpretation of shared knowledge by each individual remains to be a question for both perspectives [16]. As a synthesized view of communication, the socio-cognitive approach integrates and balances the two philosophical views. This approach to intercultural pragmatics reiterated by [16] is discourse segmented-centered rather than utterancecentered as typified by traditional pragmatic theories.

Reference [16] proposes that the socio-cognitive approach as a theoretical framework to intercultural pragmatics is set in the context of two important claims. The first one is the equal relationship between the speaker and the hearer in the communicative process. They both "produce and comprehend, while relying on their most accessible and salient knowledge as expressed in their private contexts in production and comprehension [16]." They are the same person with the same knowledge and comprehensibility when producing and interpreting language. The differences lie in their goals and functions. The socio-cognitive approach thus provides a holistic interpretation of human communication from both the speaker's perspective and the hearer's perspective. The second claim is the dynamic nature of communication, which is featured by the interplay of two inseparable, mutually supportive and interactive sets of traits - societal traits and individual traits as shown in "Table I". These traits are in sequentially causal relations to each other. That is, each trait as listed in the chart is the consequence of others. For example, prior experience results in salience, which in turn leads to egocentrism and then attention.

TABLE I. The Two SETS OF TRAITS IN SCA By KeCSKES

\begin{tabular}{l|l}
\hline \multicolumn{1}{c|}{ Societal traits } & \multicolumn{1}{c}{ Individual traits } \\
\hline actual situational experience & prior experience \\
relevance & salience \\
cooperation & egocentrism \\
intention & attention \\
\hline
\end{tabular}

To specify, intention is cooperation-directed and attention is egocentrism-governed. Attention is paid to others' intention, the presumed and commonly shared perception. The relationship between attention and intention in socio-cognitive approach is viewed as "dialectical" [16] rather than isolated. Intention is the reason or goal behind communication [22], and comprises two aspects: priori intention and emergent intention [16]. Attention, on the other hand, refers to the cognitive resources that pop up to communicators and ensures the consciousness of the communicative process [23]. In the course of communication, intention sets the destination while attention steers the wheel along the road. Three factors are involved in the process of highlighting the needed knowledge, mainly including interlocutors' knowledge based on prior experiences, the level of conventionality of knowledge concerned in the actual situation, and the communicators' mental state and/or the availability of attentional resources [16]. The interplay of the three factors will lead to the availability of the most salient knowledge and thus the possible occurrence of successful communication. In SCA, egocentrism does not denote any negative connotation, but represents the actions interlocutors perform under the most salient information that occur to their mind in the actual situated context.

In the socio-cognitive paradigm, individuals are not only constrained by preexisting societal conditions but also conversely shape them at the same time. Contexts are not strictly objective settings or decisive attributes, but rather "(inter)subjective constructs designed and ongoingly updated in interaction by participants as members of groups and communities [19]." Social situation per se hardly influences or is influenced by discourse, but how participants take part in and define such a situation matters.

Academic writing represents some positive or less positive features of communication, such as cooperation, rapport and politeness, pertinent to writers' self-evident desire for successful communication. As for the productive process of a writer, s/he should conform to the collectively formulated writing conventions, while at the same time showing personal stamps via linguistic expressions and ways of discourse organization. The collective and preexisting writing conventions are privatized by writers in actual situational contexts with the development of their actual writing process. The privatization witnesses individuals' 
blending their prior experiences with the actual situational contexts, and involves the personalized understanding of shared beliefs [16]. This line of thinking is partly proved by [24] study on German scientific writers' reproductive writing, where the quality and quantity of references in texts are attributed to the influence of the situational context, the immediate text production situation and writers' individual constraints, such as knowledge, skills, cultural socialization and so on. This study substantiated the socio-cognitive nature of academic writing, while the two aspects were viewed as combined rather than dynamically integrated and complemented to each other. Writers, in fact, are not citing, or writing in general, with bounded hands and thoughts by their prior experiences with the discipline unilaterally, but actively bring up the salient information to the needed attentional level in their production process.

Foci in the current research are thus on both the societal and cognitive factors, which are almost completely ignored in most recent literature [15], specified as the decisive role of preexisting context, shared conventions or norms, communicative purposes and writers' willingness to cooperate, together with the role of the individual's prior experience, existing knowledge and egocentrism.

\section{Methodology AND PROCEDURE}

Since an emic approach could pursue citation practices through the informants' eyes and contribute to providing "insider accounts," the proper way of eliciting data would be the qualitative interview [25]. The current study, therefore, employs naturalistic case study to obtain a thick description of a perplex issue in an intercultural context [26].

Introspective methods, an umbrella term for various ways of eliciting self-reflections, are adopted to collect information about participants' inconspicuous mental processes, such as feelings, thoughts, attitudes and motives [26]. Following [26] recommendation, four points are taken into consideration in the research design to improve the quality of the introspective data. 1) Participants are encouraged to write process logs and note down their ideas while writing, aiming to eliminate the negative consequences caused by time lapse exceeding two days. 2) Rich contextual information and stimuli, such as writers' developing texts and cited sources, are added to the interviews to elicit rich data. 3) The recall of directly retrievable information is encouraged during the interview. The researcher usually starts the conversation with questions like "Could you tell me something about your writing experience this week" or "What were you thinking of at this point." As [27] pointed out that a retrospective interview does not require much training because simple instructions and a direct model are often enough. The only thing researchers need to be careful with is not to cue the respondents and affect their responses. 4) The retrospective interview is conducted in the participants' first language, that is, Chinese.

\section{A. Participants}

All the three participants (see Table 2), Lily, Cathy and Steve (pseudonyms), are Mandarin Chinese speakers and currently enrolled in doctoral programs in a major university in eastern China. They major in social security, public administration, and applied chemistry respectively, representing humanities and hard sciences. All of them have been learning English for more than a decade. Lily has been learning English for more than eighteen years since primary school. Cathy and Steve had a shorter language training experience and learned English for about fourteen years since junior high school. Two of the participants, Lily and Steve, have received training in academic English, an optional course, in their Master's programs. When they entered their doctoral programs, academic English course became compulsory and was a one-semester course including academic writing and listening.

\section{B. Data Collection}

Text data and interview scripts were collected in consecutive seven months. The frequency of interviews is of individual differences caused by participants' asynchronic writing progress and logistical reasons. A background survey via semi-structured interviews was conducted for each participant to collect some basic information, and at the same time process logs (based on [28]), text-based interviews, the developing texts and the sources cited were recorded as well. The surveys, logs and interviews were collected and prepared in bilingual forms.

\section{Data Analysis}

The study primarily relied on participants' self-initiated reflections on their citing practices. The tape-recorded interviews were carefully transcribed in Chinese and partly translated into English. The transcribed texts of corresponding interview and the relevant information of the cited sources were added to each citation practice in the form of annotations. Then the data were analyzed through a recursive process [29] to focus on themes, such as participants' attitudes, motives and self-initiated strategies The coding scheme mainly followed the binary classification of individual and social traits proposed by [16], of which factors related to prior experience, salience, egocentrism and attention were distributed to individual or cognitive factors, and factors connected with actual situational experience, relevance, cooperation and intention were included in social factors.

\section{FINDINGS}

Just as the shift of composition studies from textual features to the process of writing itself [30], researchers of various philosophical and methodological orientations accordingly investigate the processes underlying the production of written texts. Citation as one of the most prominent features of academic writing can be better examined as a process under the influence of both societal and individual factors, which are inseparable, mutually supportive and interactive. As [15] states,

Socio-cognitive approach integrates the pragmatic view of cooperation and the cognitive view of egocentrism and emphasizes that both cooperation and egocentrism are 
manifested in all phases of communication, albeit to varying extents. While cooperation is an intention-directed practice which may be measured by relevance, egocentrism is an attention-oriented trait which is measured by salience. Intention and attention are identified as two measurable forces that affect communication in a systemic way. (p. 57)

A number of social factors, in corresponding to the social traits in SCA, contribute to the participants' citing behavior, namely different citing formats in Chinese and English academic writing, different disciplinary citing conventions, the influence of various readers, and their citing motivations.

First, Lily and Cathy, probably with more accessibility to Chinese literature and Chinese academic training, expressed their worries about English writing convention. They were heavily influenced by their ways of writing academic articles in Chinese. It could partly attribute to their disciplinary features that their focus was often on Chinese society and most sources they cited were written in Chinese. Though equipped with English writing style manual (APA manual), they stated their uncertainty about the writing conventions for both Chinese and English academic writing.

Second, disciplinary citing conventions are another important factor for the participants. Compared to the detailed and sophisticated citing practices in Lily's and Cathy's articles, Steve only cited in his introductory part with numbers assigned to a particular source rather than the combination of the names of the authors and dates as usually observed in articles in humanities. To cite Steve's article as an example:

To overcome the above drawbacks, the use of solid acid catalysts has got the first attention, and various catalysts like zeolites [3]-[4], heteropolyacids [5]-[6], and ionic exchange resins [7]-[9] join the research in quick succession. (Steve's third draft)

Third, the influence of potential readers is also important. Academic supervisors are usually the first readers of the participants' drafts. Journal reviewers are also gatekeepers for novices. As Cathy stated that she wanted to appeal to the journal reviewers when she submitted her revised article after modification. Accordingly, she highlighted the part, the Chinese fertility restrictions system, which she thought would be eye-catching to the reviewers.

Fourth, in general, writers employ citation practices to negotiate with scholars in their academic circles and hence expected to use their shared framework, rhetorical structure and linguistic expressions. Citing, as an important means to positioning, is a conscious, dynamic, and purposeful choicemaking process. Reference [31] was one of the first researchers who systematically and directly elicit the motivations of specific citations by academic writers. His interview-based results represented a wide spectrum of disciplines (fifteen altogether, e.g., anatomy, computer science, and education), and with a factor analysis to the original seven citer motives Brooks classified writers' motivations into three groups: 1) persuasiveness, positive credit, currency, and social consensus; 2) negative credit; and 3) reader alert and operational information.
Data collected from the three participants as mentioned above exemplified all these three points. They cited sources for the purpose of building their academic credibility and providing background information. In particular, Steve cautiously handled the points he disagreed with, and avoided negative words to alleviate possible negative intention.

Citation practice is individualized and subjective to some extent. It is a highly conscious action and endows the writers with great freedom. According to [16], individual factors/traits include prior experience, salience, egocentrism and attention, of which attention is a key term referring to "those cognitive resources available to interlocutors that make communication a conscious action [15]." Writers' prior experience and actual situational experience may work together to affect their citing behavior.

\section{CONCLUSION}

Novices may privatize their citation practices in academic writing by blending prior experiences and actual situational experiences. The prerequisite perception, or their motivations of citing, is something that writers bear in mind prior to their writing practices. However, in the course of writing, the emergent or social nature of original intention may override its preexisting nature due to the dynamicity of intention per se. The selection and way of organizing the cited sources are confined by not only the actual writing context, but also by writers' privatized way of citing behavior. Writers, as represented in the cases, may actively take part in the process, such as choosing the favorable sources to build up their professionalism and cautiously being cooperative with their potential readers, to leave a personal stamp on their citations, both as a process and as a product.

Writing is a collection of durably stored utterances, essentially different from most talks. Possible misunderstanding or miscommunication may lie in writers' ignorance and insufficient prediction of the actual context. Novices are even more likely to do so.

Novice EFL writers are positioning themselves in an English academic conventional system, which is reported to be different from their domestic one at times, when they write for international publication. This type of actual situational experience may be brand-new and at the same time quite challenging to them. However, the current research revealed that novices were not passively accept the changes or the strangeness, rather they were actively privatized the citing process with the help of their prior experiences and current inflections, their ability to represent the relative important information via linguistic or discoursal realization, and the involvement of their total consciousness.

Cultural difference or influence, an important issue in intercultural domain, is not discussed here partly because its influence on writers' citation practices remains questionable. It would be too simplistic to take cultural differences as a determining factor to account for novices' citation practices. When asked, "Do you find any differences while writing English academic papers compared with your Chinese writing?", the three participants answered unanimously that 
copying without acknowledgement is regarded as immoral in both Chinese and English cultures, which is in accordance with the studies of [10] and [11], who wrote about Chinese and Vietnamese perceptions of plagiarism respectively.

The interplay of societal and cognitive factors plays a crucial role in novice writers' citation practices. This is definitely a topic deserving further exploration. The current study supplemented the application of socio-cognitive approach on language use in written setting, one of the main foci of intercultural pragmatics. It further testified the application of SCA to academic writing, citation practice in specific. Reference [16] mentioned conversational analysis, discourse segment analysis (including discourse segmentation analysis combined with $\mathrm{CA}$ and centering theory), corpus analysis, computer-mediated communication are the major methods of analysis in intercultural pragmatics. Considering the written setting defined by the research topic and the insignificance of data in term of quantity, the current study initiated from case tracking, and the collected data were then analyzed via a recursive process to notice the trends or patterns regarding sets of themes.

\section{REFERENCES}

[1] J. P. Gee, Social Linguistics and Literacies: Ideology in Discourses (3rd ed.), Abingdon: Routledge, 2008.

[2] D. Pecorari, and P. Shaw, "Types of student intertextuality and faculty attitudes," Journal of Second Language Writing, vol. 21, pp. 149-164, 2012.

[3] B. Petrić, and N. Harwood, "Task requirements, task representation, and self-reported citation functions: An exploratory study of a successful L2 student's writing", Journal of English for Academic Purposes, vol. 12(2), pp. 110-124, 2013.

[4] J. Flowerdew, and Y. Li, "Plagiarism and second language writing in an electronic age", Annual Review of Applied Linguistic, vol. 27, pp. $161-183,2007$.

[5] K. Hyland, Disciplinary Discourses: Social Interactions in Academic Writing, Ann Arbor: University of Michigan Press, 2004

[6] A. Lyon, "You Fail': Plagiarism, the Ownership of Writing, and Transnational Conflicts", College Composition \& Communication, vol. 61(2), pp. 222-239, 2009.

[7] D. Pecorari, "Visible and occluded citation features in postgraduate second-language writing”, English for Specific Purposes, vol. 25(1), pp. 4-29, 2006.

[8] A. Pennycook, "Borrowing others' words: Text, ownership, memory, and plagiarism”, TESOL Quarterly, vol. 30, pp. 201-230, 1996.

[9] C. Sowden, "Plagiarism and the culture of multilingual students in higher education abroad", ELT Journal, vol. 59(3), pp. 226-233, 2005 .

[10] D. Liu, "Plagiarism in ESOL students: Is cultural conditioning truly the major culprit", ELT Journal, vol. 59(3), pp. 234-241, 2005.

[11] L. Phan, "Plagiarism and overseas students: stereotypes again?", ELT Journal, vol. 60(1), pp. 76-78, 2006.

[12] G. Yu, "Wenxian yinyong xingweizhong pipanxing siwei de gean yanjiu [A case study of critical thinking in citation behavior]", Waiyu Xuekan [Foreign Language Research], vol. 5, pp.124-128, 2007.

[13] F. Hyland, "Dealing with plagiarism when giving feedback", ELT Journal, vol. 55(4), pp. 375-381, 2001.

[14] I. Klitgård, "From copy-and-paste to trace-and-learn: A qualitative survey of student perceptions of plagiarism", Zeitschrift Schreiben, pp. $1-7$. Retrieved from: http://www.zeitschriftschreiben.eu/Beitraege/klitgard_Qualitative_Survey.pdf, 2009.
[15] I. Kecskes, "The paradox of communication: Socio-cognitive approach to pragmatics", Pragmatics \& Society, vol. 1, pp. 50-73, 2010.

[16] I. Kecskes, Intercultural Pragmatics, New York: Oxford University Press, 2014.

[17] D. Atkinson, "Toward a sociocognitive approach to second Language acquisition," The Modern Language Journal, vol. 86, pp. 525-545, 2002.

[18] T. Ringberg, and R. Markus, "Towards a socio-cognitive approach to knowledge transfer", Journal of Management Studies vol. 45(5), pp. $912-935,2008$

[19] T. A. van Dijk, Discourse and Context: A Sociocognitive Approach, Cambridge: Cambridge University Press, 2008.

[20] L. Wittgenstein, Philosophical Investigations, Edited by Anscombe Gertrude Elizabeth Margaret and Rush Rhee. Translated by Anscombe Gertrude Elizabeth Margaret, Oxford: Blackwell, 1953.

[21] H. Garfinkel, Studies in Ethnomethodology. Englewood Cliffs, NJ: Prentice-Hall, 1967.

[22] J. R. Searle, Intentionality: An Essay in the Philosophy of Mind, Cambridge: Cambridge University Press, 1983.

[23] I. Kecskes, and F. Zhang, "Activating, seeking and creating common ground: A socio-cognitive approach.”, Pragmatics and Cognition, vol. 17 (2), pp. 331-355, 2009.

[24] E. Jacobs, "Reproductive writing — writing from sources", Journal of Pragmatics, vol. 35, pp. 893-906, 2003.

[25] N. Harwood, "An interview-based study of the functions of citations in academic writing across two disciplines", Journal of Pragmatics, vol. 41(3), pp. 497-518, 2009.

[26] Z. Dörnyei, Research Methods in Applied Linguistics: Quantitative, Qualitative, and Mixed Methodologies, Oxford: Oxford University Press, 2007.

[27] S. M. Gass, and M. Alison, Stimulated Recall Methodology in Second Language Research, Mahwah, NJ: Erlbaum, 2000.

[28] Y. Li, "Apprentice scholarly writing in a community of practice: An intraview of an NNES graduate student writing a research article", TESOL Quarterly, vol. 41(1), pp. 55-79, 2007.

[29] K. M. Bailey, "What my EFL students taught me",The PAC Journal, vol. 1, pp. 7-31, 2001

[30] P. K. Matsuda, "Second language writing in the twentieth century: A situated historical perspective", In Exploring the Dynamics of Second Language Writing, edited by Barbara Kroll, pp. 15-34, New York: Cambridge University Press, 2003.

[31] T. A. Brooks, "Private acts and public objects: An investigation of citer motivations", Journal of the American Society for Information Science, vol. 36, pp. 223-229, 1985. 